\title{
Attitude Control System for Directional Drilling Bottom Hole
}

\author{
Assemblies \\ Neilkunal Panchal ${ }^{1}$, Martin T. Bayliss ${ }^{2}$, and James F. Whidborne ${ }^{1}$ \\ ${ }^{1}$ School of Engineering, Cranfield University, Bedfordshire, MK43 OAL, UK. \\ n.panchal|j.f.whidborne@cranfield.ac.uk \\ ${ }^{2}$ Schlumberger, Stonehouse Technology Center, Brunel Way, Stroudwater Business Park, Gloucestershire, \\ GL10 3SX, UK. mbayliss@slb.com
}

July 19, 2011

\begin{abstract}
A general approach for the attitude control of directional drilling tools for the oil and gas industry is proposed. The attitude is represented by a unit vector, thus the non-linearities introduced by Euler angle representations are avoided. Three control laws are proposed, and their stability is proven. Their behaviour is tested by numerical simulation. The merits of the laws from an engineering perspective are highlighted, and some details for the implementation of the laws on directional drilling tools are provided.
\end{abstract}

\section{Introduction}

In the oil and gas industry, geometric boreholes are produced by the process of directional drilling. This involves steering a drilling tool in a desired direction along a path defined by a multidisciplinary team of: reservoir engineers, drilling engineers, geosteerers and geologists amongst others. Most wells drilled nowadays are horizontal wells, which consist of a vertical part, a curved part known as a build section, and a horizontal section which is steered with respect to geological features in order to maximize oil recovery from a reservoir $[1,2,3]$. The technology which enables the steering of the drill allows for turn radii as low as 120 metres $\left(15^{\circ} / 100 \mathrm{ft}\right)$, enabling complex three dimensional wells to be drilled. Directional drilling can be achieved by either Rotary Steerable Systems (RSS) $[4,5]$ and conventional slide directional drilling approaches [4, 6]. For the case of RSS directional drilling tools the Bottom Hole Assembly (BHA) lies inside the borehole and is connected to the surface by a series of steel tubular pipes collectively referred to as the drill string. A schematic of the main RSS directional drilling system components is shown in Figure 1. The drill string runs all the way to the derrick at the surface where it is suspended by a cable, and rotated by a top-drive which provides torque to the bit, hence the drill string and BHA can be viewed 


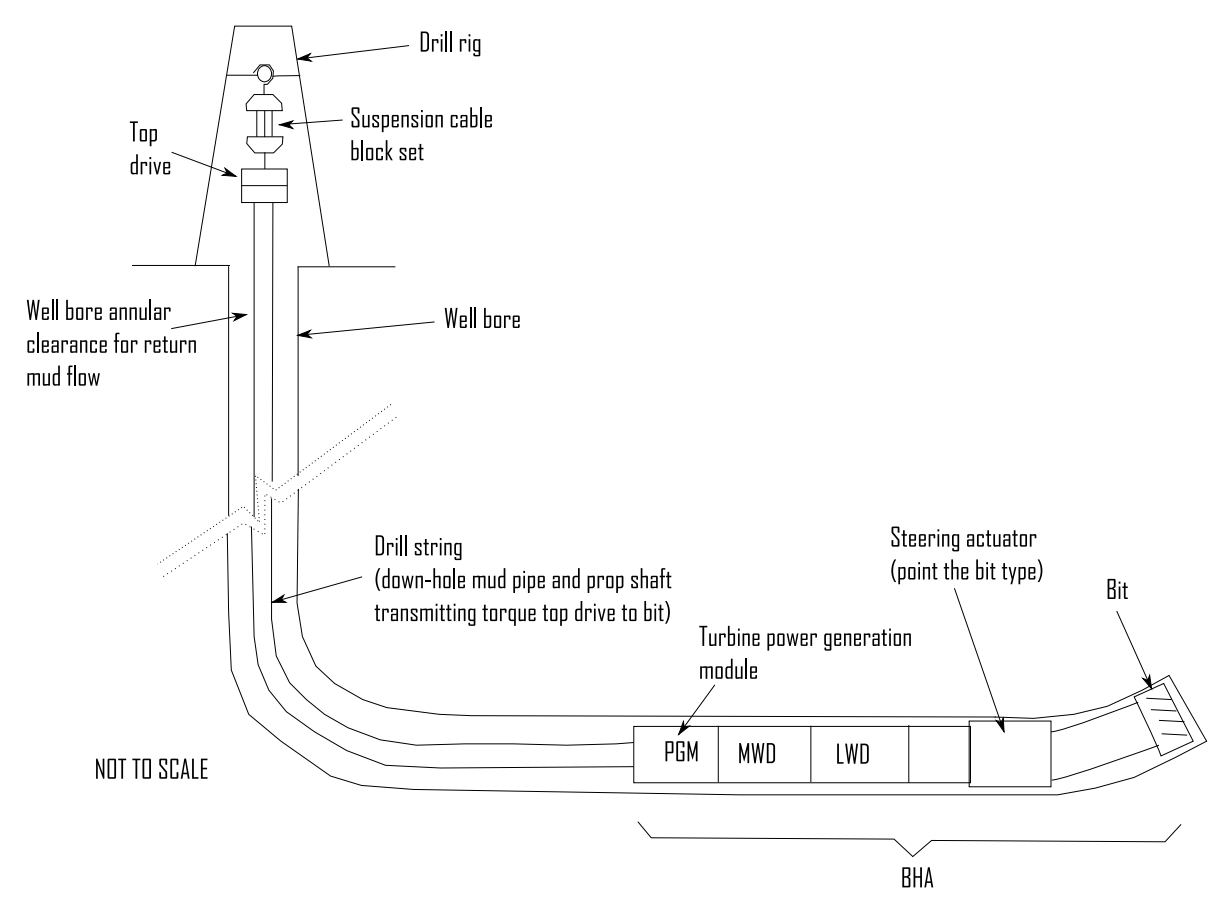

Figure 1: Schematic of main RSS directional drilling system components

as a propeller shaft delivering torque to the bit directly. Slide directional drilling is similar except the torque to drive the bit is generated downhole by a mud motor (Moyno motor) where most of the drill string and $\mathrm{BHA}$ is non-rotating relative to the formation. The $\mathrm{BHA}$ is the active part of the directional drilling system and is made up of subsystems or 'subs' from the bit back to the first drill pipe of the drill string. The subs that constitute the BHA are configured to suit the well plan and drilling objectives, and always include a steering unit which will either be push- or point-the-bit [7] to propagate the borehole.

The subs include items such as a power generation module, Measurements While Drilling (MWD) sub, Logging While Drilling (LWD) sub, and steering unit (which includes the tool-face actuator and bit). The MWD is a sensor pack that senses attitude, collar angular velocity and position information, whereas LWD are sensor packages that sense petrophysical information such as formation resistivity, porosity and gamma-ray imaging. The important parts of the BHA from a steering perspective are the steering unit and the MWD. Most of the following discussion of directional drilling sensor practice is applicable to both RSS and sliding directional drilling tools.

It is common in directional drilling to have an outer attitude control loop which generates set point tool-face commands (either automatically or manually by the operator in the loop who is known as the directional driller) which are passed to an inner loop that controls the steering unit. For the outer attitude control loop the attitude sensor set consists of one triaxis set of accelerometer and one triaxis set of magnetometer transducers, both arranged with the same orientation and sign convention. All six transducer signals are then used to evaluate the orientation azimuth $\theta_{a z i}$ and inclination $\theta_{i n c}$ (see Figure 2) of the MWD, which is mechanically continuous with the steering unit. In this paper the tool and global coordinate system used is a right handed coordinate system with the $x$-axis pointing down-hole 
towards the bit and down respectively.

However, for measuring the actuator tool-face for the inner actuator tool-face control loop, it is only necessary to use either the radial magnetometer or accelerometer signals where, in this context, the actuator tool-face is defined to be the angular position of the resultant force applied by the steering unit onto the formation. The angular position tool-face of the resultant force is measured relative to the projection of the magnetic or gravitational field vector onto a plane at right angles to the BHA. The angular position for the former is known as the Magnetic tool-face (MTF) whereas the latter is known as the Gravity tool-face (GTF) (see figure 2).

It is common to define a well plan as a series of gravity tool-face values since they are easier to visualize for the directional driller (up is $0^{\circ} \mathrm{GTF}$, down is $180^{\circ} \mathrm{GTF}$, right is $90^{\circ} \mathrm{GTF}$ and left is $270^{\circ} \mathrm{GTF}$ ). Some tools can only actuate their tool-face in MTF because they use strap down control actuator units where measuring tool-face directly with the radial accelerometers is not possible as the sensors rotate with the drill string collar and filtering the radial signals to remove the collar rotational component introduces destabilizing lags into any control system utilizing these signals. In this situation, based on measuring the difference in the static survey MTF and GTF it is possible to evaluate the MTF whilst the tool is drilling which is equivalent to the GTF the directional driller wishes to apply.

For strap down or roll stabilized sensor actuated drilling tools, it is the case that the MWD sensor pack is used to make attitude measurements both continuously when the tool is sliding or rotating and statically when the tool is not propagating. This applies to RSS and non-RSS tools. The former is known as continuous surveying and the latter is known as a static survey. Because of the accelerometers the static surveys are always much more accurate and are used to measure other quantities such as the magnetic and gravitational field strengths and the magnetic field dip angle. These quantities are then used in subsequent continuous surveys where just by using the continuous axial accelerometer and magnetometer signals combined with these static survey quantities the continuous survey azimuth and inclination measurements can be made. These continuous attitude measurements therefore become less accurate the further the tool propagates from the position where the static survey was taken. This approach for continuous surveying only being possible due to the low curvature responses of most drilling tools relative to their rate of propagation. Hence in practice directional drillers periodically stop drilling to make accurate static surveys so as to improve the accuracy of the continuous surveys.

It can be appreciated that in practice the MWD used for attitude measurement are by necessity some distance (sometimes several tens of feet) back from the steering unit for which the attitude measurement is being made. This introduces a significant measurement delay in the attitude feedback measurement which any outer attitude control loop should be robust enough to deal with in terms of stability and performance. Additionally there can be a significant dynamic response between the applied tool-face from the actuator and the response tool-face of the steering unit.

In this paper, the attitude measurement and tool-face actuation are not discussed in detail but the preceding discussion has been included to put the subsequent work into a directional drilling context.

The objective for the directional-drilling attitude control system is to hold an attitude specified by 


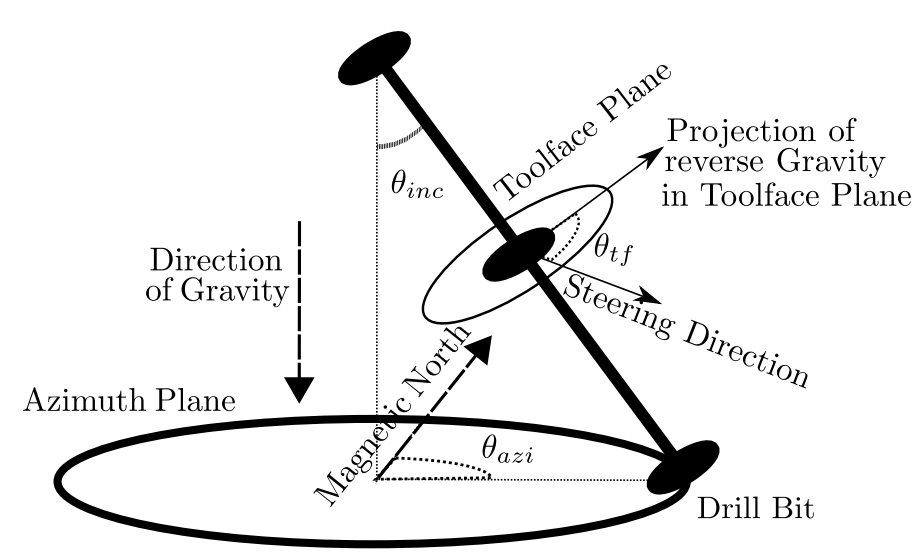

Figure 2: Conventional attitude and steering parameters for a Bottom Hole Assembly(BHA)

inclination and azimuth angle set points [8]. The inclination $\theta_{i n c}$ and azimuth $\theta_{a z i}$ angles are shown in Figure 2. These set-points are communicated to the BHA via low bandwidth ( 1 to 5 bits per second) mud pulse telemetry. The attitude control described in this paper is intended to be general and applicable to both RSS and sliding directional drilling for push or point the bit steering units.

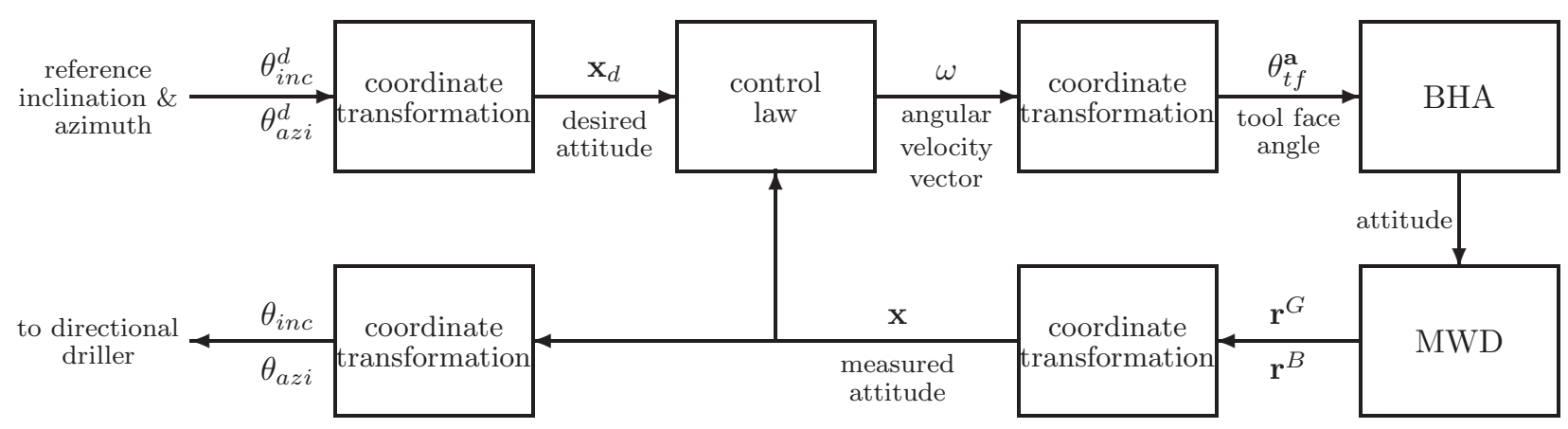

Figure 3: System structure

The attitude control system proposed in this paper is depicted in Figure 3. Here the directional driller would send attitude reference signals to the drill, these being inclination $\theta_{i n c}^{d}$ and azimuth $\theta_{a z i}^{d}$ set points of the desired tool orientation in the Earth reference frame. These demand inclination and azimuth signals are converted to a unit vector in the $R^{3}$ vector space, representing the drill's attitude. In the next section, the model for the drill dynamics is presented. In Section 3, three control laws are proposed that stabilize the attitude and drive it towards the set point. The stability of the proposed laws are proven using the direct method of Lyapunov. Section 4 presents details about the coordinate transformations necessary for implementing the proposed controllers in line with existing convention for the drilling industry. Some simulation results are presented in Section 5, and in the final section some conclusions are drawn and suggestions given for further work. 


\section{Kinematic model of the drill}

The BHA is modeled as a rigid rod hinged at one end that has only the rotational motion corresponding to what can be interpreted as pitch and yaw, with the roll motion ignored. The rotation rates are small and the translational kinetic energy is assumed zero. Furthermore, the motion of the BHA is constrained by the well and hence momentum terms are redundant. Hence the kinematic system representing the time varying response of the tools attitude [9] can be represented as

$$
\dot{\mathbf{x}}=\omega \times \mathbf{x}
$$

where $\mathbf{x} \in \mathbb{R}^{3}$ is a unit vector representing the tools attitude and $\omega \in \mathbb{R}^{3}$ is the angular velocity vector parameter (the magnitude of which is referred to here as the build rate). Given an initial value, $\mathbf{x}(0)=x_{0}$, $\left\|\mathbf{x}_{0}\right\|=1$, and a control $\omega$, the resulting trajectory, $\mathbf{x}(t)$ lies on the surface of the unit sphere, that is $\|\mathbf{x}(t)\|=1$ for all $t$ and $\omega$. The kinematic motion is controlled by varying $\omega$ via the tool-face angle (see section 4.4 for details). Note that (1) can be expressed as:

$$
\dot{\mathbf{x}}=M \mathbf{x}
$$

where

$$
M=\left(\begin{array}{ccc}
0 & -\omega_{3} & \omega_{2} \\
\omega_{3} & 0 & -\omega_{1} \\
-\omega_{2} & \omega_{1} & 0
\end{array}\right) \quad \text { and } \quad \omega=\left(\begin{array}{c}
\omega_{1} \\
\omega_{2} \\
\omega_{3}
\end{array}\right)
$$

The BHA is subject to disturbances due to varying rock formations. In addition there is a tendency for the BHA to drop towards a vertical orientation due to gravity, and a tendency for the BHA to drift horizontally. The disturbances are ignored in the model.

The model also ignores dynamics of the actuator. The tool-face response is subject to lags, however for most tools (though not all) these are generally of a much higher bandwidth than the model kinematics and, as for this paper, can be ignored.

\section{Attitude Control Laws and Stability}

In this section, three control laws are proposed for the control of the tool attitude. The stability of the laws is proved by means of Lyapunov direct method using a lemma that is derived directly from the Lyapunov Theorem of Local Stability [10]. The following definition [10] is first required.

Definition 1. An equilibrium point $\tilde{\mathbf{x}}$ is a state of the system (1) such that when $\mathbf{x}(t)$ is equal to $\tilde{\mathbf{x}}$, it remains so for all subsequent times. That is $\dot{\mathbf{x}}=0$.

Lemma 1. For a dynamical system to be locally stable, there exists a scalar valued function $V(\mathbf{x})$ with continuous first partial derivatives in a neighbourhood $B$ about the equilibrium point such that:

- $V(\mathbf{x})$ is positive definite, 
- $\dot{V}(\mathbf{x})$ is negative semi-definite.

In addition, if the derivative $\dot{V}(\mathbf{x})$ is negative definite in $B$, then the stability is asymptotic.

\subsection{Variable build-rate controller}

Proposition 1. The dynamical system given by (1) with the feedback control law

$$
\omega=k \mathbf{x} \times \mathbf{x}_{d}
$$

is locally asymptotically stable at the equilibrium point $\mathbf{x}=\mathbf{x}_{d}$ for $\mathbf{x} \in B$, for all $k \in \mathbb{R}^{+} \backslash\{0\}$ where $\mathbf{x}_{d} \in \mathbb{R}^{3}$ is the demand attitude of the tool, and where

$$
B:=\left\{\mathbf{x}:\|\mathbf{x}\|=1 \text { and } \mathbf{x} \in \mathbb{R}^{3} \text { and } \mathbf{x} \neq-\mathbf{x}_{d}\right\}
$$

Proof. For the tool to converge towards its demand attitude $\mathbf{x}_{d}$, this must be an equilibrium point. The neighbourhood $B$ around the equilibrium point is defined to be the set of all attitudes $\mathbf{x}$ such that $\mathbf{x} \neq-\mathbf{x}_{d}$. Substituting the feedback control law (4) into the attitude dynamics (1) gives

$$
\dot{\mathbf{x}}=k\left[\mathbf{x}_{d}-\left(\mathbf{x} \cdot \mathbf{x}_{d}\right) \mathbf{x}\right] .
$$

Setting $\mathbf{x}=\mathbf{x}_{d}$ in (6) gives $\dot{\mathbf{x}}=0$, and hence by Definition 1 this is an equilibrium point. We use the Lyapunov function

$$
V(\mathbf{x})=\frac{1}{2}\left[1-\left(\mathbf{x} \cdot \mathbf{x}_{d}\right)^{2}\right] .
$$

Here $V\left(\mathbf{x}=\mathbf{x}_{d}\right)=0$ and for all $\mathbf{x} \in B, V(\mathbf{x})>0$, since $\mathbf{x}$ and $\mathbf{x}_{d}$ are unit vectors. The first derivative of the Lyapunov function is given by

$$
\begin{aligned}
\dot{V}(\mathbf{x}) & =-\dot{\mathbf{x}} \cdot \mathbf{x}_{d} \\
& =-(\omega \times \mathbf{x}) \cdot \mathbf{x}_{d} \\
& =-\left[\mathbf{x}_{d}-\left(\mathbf{x}_{d} \cdot \mathbf{x}\right) \mathbf{x}\right] \cdot \mathbf{x}_{d} \\
& =-\left[1-\left(\mathbf{x}_{d} \cdot \mathbf{x}\right)^{2}\right]
\end{aligned}
$$

which is negative definite and hence by Lemma 1 the system (1) with control law (4) is locally asymptotically stable.

The control law given by (4) is remarkably simple and elegant. Similar control laws are proposed in [9]. The control law is linear and avoids the non-linearities associated with Euler angle representations such as the controller proposed in [7].

\subsection{Constant build-rate controller}

The controller assumes that the build rate is proportional to the attitude error. However, in practice, this is very difficult to engineer. For real directional drilling BHA, the actuator build rate is generally a 
constant. Hence a second control law is proposed where the assumption of a continuously variable build rate is removed and it is assumed that the build rate is constant or zero.

Proposition 2. The dynamical system given by (1) with the feedback control law

$$
\omega= \begin{cases}K \frac{\mathbf{x} \times \mathbf{x}_{d}}{\left\|\mathbf{x} \times \mathbf{x}_{d}\right\|} & \text { for } \mathbf{x} \neq \mathbf{x}_{d} \\ 0 & \text { for } \mathbf{x}=\mathbf{x}_{d},\end{cases}
$$

is locally asymptotically stable at the equilibrium point $\mathbf{x}=\mathbf{x}_{d}$ for $\mathbf{x} \in B$ where $K$ is the constant build rate.

Proof. From (9) by definition $\mathbf{x}=\mathbf{x}_{d}$ is an equilibrium point. We again use the Lyapunov function given by (7). The first derivative of the Lyapunov function is given by

$$
\dot{V}(\mathbf{x})= \begin{cases}\frac{-K\left[1-\left(\mathbf{x}_{\mathbf{x}}\right)^{2}\right]}{\left\|\mathbf{x} \times \mathbf{x}_{d}\right\|} & \text { for } \mathbf{x} \neq \mathbf{x}_{d} \\ 0 & \text { for } \mathbf{x}=\mathbf{x}_{d}\end{cases}
$$

which is negative definite and hence by Lemma 1 the system (1) with control law (9) is locally asymptotically stable.

Corollary 1. The control give by (9) solves the minimum time optimal control problem defined as

$$
\begin{aligned}
& \min _{\omega} \int_{0}^{t_{f}} d t \\
& \text { subject to }(1), \mathbf{x}(0)=x_{0},\left\|\mathbf{x}_{0}\right\|=1, \mathbf{x}\left(t_{f}\right)=\mathbf{x}_{d},\|\omega\| \leq K .
\end{aligned}
$$

Proof. Let $\hat{\mathbf{x}}(t)$ be the trajectory that is the solution to the initial value problem for system (1) with the control given by (9) and $\mathbf{x}(0)=\mathbf{x}_{0},\left\|\mathbf{x}_{0}\right\|=1$. The tangent to the curve $\hat{\mathbf{x}}(t)$ is given by

$$
\omega \times \mathbf{x}=K \frac{\mathbf{x}_{d}-\mathbf{x}\left(\mathbf{x} \cdot \mathbf{x}_{d}\right)}{\left\|\mathbf{x} \times \mathbf{x}_{d}\right\|}
$$

Since the tangent vector is spanned by the vectors $\mathbf{x}_{d}$ and $\mathbf{x}$ which pass through the origin on the sphere, the state trajectory $\hat{\mathbf{x}}(t)$ is a geodesic. Geodesics on a sphere are arcs of great circles with the property that they are the intersection of the sphere and a plane passing through the origin, where the great circle lies on this plane. It is well-known that a geodesic gives the minimum distance over the surface between two points lying on that surface [11, p. 218]. Clearly, the path of minimum length is the path of the minimal time trajectory if the speed along the path is always maximal. From (9), the build rate is maximum (except when the target is reached), hence the trajectory is minimum time.

The control law given by (9) assumes that build rate can be zero. In practice, the build rate can generally not be made zero and rectilinear boreholes are achieved through spinning $\omega$ about the $\mathbf{x}$-axis. In addition, small perturbations on the system could cause the control to switch very rapidly about the desired attitude. This chattering behaviour is also well known to occur with sliding mode controllers switching about the sliding manifold [12]. Furthermore, the controllers are usually implemented with a digital processor. By implementing the control law in the discrete time domain, the chattering problem can be reduced. The final proposed controller is thus a discrete time controller with a similar control law to $(9)$. 


\subsection{Discrete-time controller}

Let us assume a constant build rate, $K$, and a constant sample time, $\delta t$. Let us denote the state at the $i^{\text {th }}$ sample time, $t=i \delta t$ by $\mathbf{x}(i)$. Define the constant $\gamma \in \mathbb{R}_{+}$to be the cosine of the angle the tool builds in one sample time, that is $\gamma=\cos (K \delta t)$. The discrete-time system for the evolution of the attitude of the penetrating drill can be modeled by

$$
\mathbf{x}(i+1)=\Gamma(\mathbf{x}(i))=\gamma \mathbf{x}(i)+\sqrt{\left(1-\gamma^{2}\right)} \tilde{\omega}(i) \times \mathbf{x}(i)
$$

where $i \in \mathbb{Z}$ is the sample number and $\tilde{\omega}$ is the normalized unit angular velocity vector. The attitude must remain a unit vector and the term $\tilde{\omega}(i) \times \mathbf{x}(i+1)$ is restricted to be perpendicular to the tool's attitude, since the actuation to steer the drill does not contribute to rate of penetration. The constant $\gamma$ is the scalar advancement in the tools actuation in one time step, that is

$$
\gamma=\mathbf{x}(i) \cdot \mathbf{x}(i+1)
$$

The constraint on the control requiring it to have a constant build rate is imposed by practical engineering considerations. It is far cheaper and simpler to engineer the actuator to have a constant build rate. For a drilling tool the value of $\gamma$ is usually greater than 0.95 ; drilling tools typically propagate through the ground at a rate of $100 \mathrm{ft} /$ hour or less with curvatures up to $15^{\circ} / 100 \mathrm{ft}$. Samples are taken about every 100 seconds.

The constant build rate constraint has implications for the stability of the closed loop system, because the system can no longer be at equilibrium when the error is zero. Hence we need to modify the stability requirements from standard asymptotic stability. Essentially, we need the error in the tool attitude to be bounded in a small region around the required attitude. Furthermore the attitude must converge to this region if it is outside it. The concept of $(I, J)$ stability [13] allows us to proceed.

Definition 2. Given a discrete-time system, $x(i+1)=\Gamma(x(i))$, and two invariant sets $J \subseteq I$ with respect to the affine map $\Gamma(x(i))$, we say that $\Gamma(x(i))$ is $(I, J)$ - stable if $\forall x(0) \in I, \exists i_{0} \geq 0: x_{i} \in J, \forall i \geq i_{0}$.

Proposition 3. The dynamical system in (13) with the controller given by

$$
\tilde{\omega}(i)= \begin{cases}\frac{\mathbf{x}(i) \times \mathbf{x}_{d}}{\left\|\mathbf{x}(i) \times \mathbf{x}_{d}\right\|} & \text { for } \mathbf{x}(i) \neq \pm \mathbf{x}_{d} \\ \tilde{\omega}(i-1) & \text { for } \mathbf{x}(i)=\mathbf{x}_{d}, i \neq 0 \\ \tilde{\omega}_{l} & \text { for } \mathbf{x}(i)=-\mathbf{x}_{d} \text { or } \mathbf{x}_{d} \text { and } i=0\end{cases}
$$

is $(I, J)$-stable, where $\tilde{\omega}_{l}$ is any unit vector satisfying $\tilde{\omega}_{l} \cdot \mathbf{x}(i)=0$

Proof. The invariant set $I$ is defined to be the set of all possible attitudes

$$
I:=\left\{\mathbf{x}(i) \in \mathbb{R}^{3}:\|\mathbf{x}(i)\|=1\right\}
$$

The set $J$ is in the neighborhood of the desired attitude $\mathbf{x}_{d}$ and is

$$
J \subseteq I:=\left\{\mathbf{x}(i) \in I: \mathbf{x}(i) \cdot \mathbf{x}_{d} \geq \gamma\right\}
$$


To show that $J$ is an invariant set, consider first the case when $\mathbf{x}(i) \cdot \mathbf{x}_{d}=\gamma$, then the case when $\mathbf{x}(i) \cdot \mathbf{x}_{d}>\gamma$. For the case $\mathbf{x}(i) \cdot \mathbf{x}_{d}=\gamma$, the proof is by induction, where it is to be shown that if $\mathbf{x}(i) \in J$ then $\mathbf{x}(i+1) \in J \forall i \in \mathbb{N}$. Substituting the control law from (15) into the dynamical system from (13) gives the closed-loop system

$$
\mathbf{x}(i+1)=\mathbf{x}_{d} \frac{\sqrt{1-\gamma^{2}}}{\sqrt{1-\left(\mathbf{x}(i) \cdot \mathbf{x}_{d}\right)^{2}}}+\mathbf{x}(i)\left[\gamma-\sqrt{1-\gamma^{2}} \frac{\mathbf{x}(i) \cdot \mathbf{x}_{d}}{\sqrt{1-\left(\mathbf{x}(i) \cdot \mathbf{x}_{d}\right)^{2}}}\right] .
$$

Taking the scalar product of (18) with $\mathbf{x}_{d}$ and substituting for $\mathbf{x}(i) \cdot \mathbf{x}_{d}=\gamma \operatorname{gives} \mathbf{x}(i+1) \cdot \mathbf{x}_{d}=1$. This corresponds to the attitude being the desired attitude $\mathbf{x}(i+1)=\mathbf{x}_{d}$. The attitude at time $i+2$ is given using $\tilde{\omega}(i)$ as the control by

$$
\mathbf{x}(i+2)=\gamma \mathbf{x}(i+1)-\frac{\sqrt{1-\gamma^{2}}}{\sqrt{1-\left(\mathbf{x}(i) \cdot \mathbf{x}_{d}\right)^{2}}}\left[\left(\mathbf{x}(i+1) \cdot \mathbf{x}_{d}\right) \mathbf{x}(i)-(\mathbf{x}(i+1) \cdot \mathbf{x}(i)) \mathbf{x}_{d}\right] .
$$

Taking the scalar product of (19) with $\mathbf{x}_{d}$ and substituting for $\mathbf{x}(i) \cdot \mathbf{x}_{d}=\gamma$ and $\mathbf{x}(i+1) \cdot \mathbf{x}_{d}=1$ gives $\mathbf{x}(i+2) \cdot \mathbf{x}_{d}=\gamma$.

For the case when $\gamma<\mathbf{x}(i) \cdot \mathbf{x}_{d}<1$ initially, take the scalar product of (18) with $\mathbf{x}_{d}$. This gives the expression

$$
\mathbf{x}(i+1) \cdot \mathbf{x}_{d}=\sqrt{1-\left(\mathbf{x}(i) \cdot \mathbf{x}_{d}\right)^{2}} \sqrt{1-\gamma^{2}}+\mathbf{x}(i) \cdot \mathbf{x}_{d} \gamma
$$

Since $\gamma=\cos K \delta t$, and $\sqrt{1-\gamma^{2}}=\sin K \delta t,(20)$ can be written in terms of the double angle formula so

$$
\mathbf{x}(i+1) \cdot \mathbf{x}_{d}=A \cos (K \delta t-\alpha)
$$

where $A=1$ and $\alpha=\arctan \left(\left(\sqrt{1-\mathbf{x}(i) \cdot \mathbf{x}_{d}}\right) /\left(\mathbf{x}(i) \cdot \mathbf{x}_{d}\right)\right)$. But since $\mathbf{x}(i) \cdot \mathbf{x}_{d}$ is a scalar product and can be written as $\cos \left(\mathbf{x}(i) \cdot \mathbf{x}_{d}\right)=\cos \alpha$, and given the condition that $\gamma<\mathbf{x}(i) \cdot \mathbf{x}_{d}<1$ implies that $0<\alpha<K \delta t$, then $0<K \delta t-\alpha<K \delta t$ and hence $\mathbf{x}(i+1) \cdot \mathbf{x}_{d}>\gamma$ and $J$ is thus an invariant set.

Now consider $\mathbf{x}(i) \in I, \mathbf{x}(i) \neq \pm \mathbf{x}_{d}$ and $\left(\mathbf{x}(i) \cdot \mathbf{x}_{d}\right)<\gamma$. Here we chose a Lyapunov function to be

$$
V(i)=1-\left(\mathbf{x}(i) \cdot \mathbf{x}_{d}\right)^{2}
$$

where $0<V<1$.

The Lyapunov function strictly decreases that is $V(i+1)<V(i)$ if the attitude converges towards the set $J$. Substituting the control law (15) into (13) and taking the scalar product of this with the demand attitude vector $\mathbf{x}_{d}$ gives

$$
\mathbf{x}(i+1) \cdot \mathbf{x}_{d}=\gamma \mathbf{x}(i) \cdot \mathbf{x}_{d}+\sqrt{1-\left(\mathbf{x}(i) \cdot \mathbf{x}_{d}\right)^{2}} \sqrt{1-\gamma^{2}}
$$

Subtracting $\mathbf{x}(i) \cdot \mathbf{x}_{d}$ from $(23)$ gives

$$
\mathbf{x}(i+1) \cdot \mathbf{x}_{d}-\mathbf{x}(i) \cdot \mathbf{x}_{d}=(\gamma-1) \mathbf{x}(i) \cdot \mathbf{x}_{d}+\sqrt{1-\left(\mathbf{x}(i) \cdot \mathbf{x}_{d}\right)^{2}} \sqrt{1-\gamma^{2}}
$$

Adding $\mathbf{x}(i) \cdot \mathbf{x}_{d}$ from (23) gives

$$
\mathbf{x}(i+1) \cdot \mathbf{x}_{d}+\mathbf{x}(i) \cdot \mathbf{x}_{d}=(\gamma+1) \mathbf{x}(i) \cdot \mathbf{x}_{d}+\sqrt{1-\left(\mathbf{x}(i) \cdot \mathbf{x}_{d}\right)^{2}} \sqrt{1-\gamma^{2}}
$$


Multiplying (24) with (25) gives

$$
\left(\mathbf{x}(i+1) \cdot \mathbf{x}_{d}\right)^{2}-\left(\mathbf{x}(i) \cdot \mathbf{x}_{d}\right)^{2}=2\left(\gamma^{2}-1\right)\left(\mathbf{x}(i) \cdot \mathbf{x}_{d}\right)^{2}+2 \gamma \mathbf{x}(i) \cdot \mathbf{x}_{d} \sqrt{1-\left(\mathbf{x}(i) \cdot \mathbf{x}_{d}\right)^{2}} \sqrt{1-\gamma^{2}} .
$$

From (22), the left hand sided of (26) can be expressed in terms of the Lyapunov function as $V(i)-V(i+1)$, The condition for this stability can be re-expressed as

$$
2\left(\gamma^{2}-1\right)\left(\mathbf{x}(i) \cdot \mathbf{x}_{d}\right)^{2}>-2 \gamma \mathbf{x}(i) \cdot \mathbf{x}_{d} \sqrt{1-\left(\mathbf{x}(i) \cdot \mathbf{x}_{d}\right)^{2}} \sqrt{1-\gamma^{2}} .
$$

simplifying gives

$$
\frac{\mathbf{x}(i) \cdot \mathbf{x}_{d}}{\sqrt{1-\left(\mathbf{x}(i) \cdot \mathbf{x}_{d}\right)^{2}}}<\frac{\gamma}{\sqrt{1-\gamma^{2}}}
$$

where this expression holds for all $\mathbf{x}(i) \cdot \mathbf{x}_{d}<\gamma$. Since $\mathbf{x}(i) \cdot \mathbf{x}_{d}$ is the angle between the attitude $\mathbf{x}(i)$ and the desired attitude $\mathbf{x}_{d}$, which are both unit vectors, then writing $\mathbf{x}(i) \cdot \mathbf{x}_{d}=\cos (\vartheta)$ for some angle $\vartheta \in[0, \pi)$, simplifies $(28)$ to

$$
\tan \vartheta<\tan K \delta t .
$$

Hence the Lyapunov function decreases in each step provided that $\vartheta>K \delta t$.

Lastly consider an initial attitude being the antipodal point $\mathbf{x}_{0}=-\mathbf{x}_{d}$. Here $\tilde{\omega}_{l}$ cannot be determined from the vector product of the current measured attitude and the desired attitude, however any direction $\tilde{\omega}_{l}$ would suffice since in the next time step, the attitude would move away from the antipodal point and a value of $\tilde{\omega}$ can be determined and the attitude will converge towards the set $J$. Furthermore, for an initial attitude beginning at the desired attitude $\mathbf{x}_{0}=\mathbf{x}_{d}$, since there is no previous time step control to apply, any control $\tilde{\omega}_{l}$ would move the attitude to $\mathbf{x}_{1} \cdot \mathbf{x}_{d}=\gamma$, since $\mathbf{x}(i) \cdot \mathbf{x}(i+1)=\gamma$, and the aforementioned argument for $\mathbf{x}(i) \cdot \mathbf{x}_{d}=\gamma$ implies $(I, J)$-stability.

\section{Controller Implementation}

Although the control laws proposed in Section 3 are simple and can be analyzed using well-known methods, several coordinate transformations are required in order to implement the controller within the structure shown in Figure 3. The attitude of the BHA has to be determined from measurements of the accelerometers and magnetometers, the desired attitude, $x_{d}$, has to be calculated as a unit vector from desired Euler angles, and finally the tool face angle to enact the calculated control, $\omega$, has to be computed. In this section, the calculations for these coordinate transformations are presented. However, it is possible to enact the control directly from the Euler angle; this is presented in Section 4.5.

\subsection{Determining the Attitude in the earth Frame from Accelerometers and Magnetometers}

In the following we use the Markley Algorithm as detailed by [14], and was used for the application of determining the attitude of a spacecraft in earth orbit from the earth's magnetic field and the line of sight to the sun. 
The earth frame is the inertial frame which is fixed, and corresponds locally to the geology in which a drilling operation would take place. It is assumed that the variation in the earth's magnetic and gravitational field over the region of an oil well is approximately Euclidean. In this frame there are two normalized reference vectors, these being the magnetic field $\mathbf{r}^{B}$ and the gravitational field $\mathbf{r}^{G}$. These are given relative to a basis in the earth frame which is easily measured and known. On the drill the accelerometers $\mathbf{b}^{G}$ and magnetometers $\mathbf{b}^{B}$ provide another basis where the magnetic and gravitational field is related to the earth frame by the transformation

$$
\begin{gathered}
q \mathbf{r}^{G} q^{-1}=\mathbf{b}^{G}, \\
q \mathbf{r}^{B} q^{-1}=\mathbf{b}^{B},
\end{gathered}
$$

where the quaternion $q$ can be determined from the Markley Algorithm [14]. From knowing $q$, the attitude of the drill in the earth frame is given by:

$$
\mathbf{x}=q^{-1} \mathbf{r}^{G} q
$$

Alternatively, the attitude can be calculated through

$$
\mathbf{x}=R_{y}\left(\theta_{i n c}\right) R_{x}\left(\theta_{a z i}\right) \mathbf{r}^{G}
$$

where

$$
\theta_{a z i}=\arctan \left(\frac{-\mathbf{b}_{z}^{B}}{\mathbf{b}_{y}^{B}}\right), \quad \theta_{i n c}=\arccos \frac{\mathbf{b}_{x}^{G}}{\left\|\mathbf{b}^{G}\right\|},
$$

and

$$
\begin{aligned}
R_{x}(\cdot): & =\left[\begin{array}{ccc}
1 & 0 & 0 \\
0 & \cos (\cdot) & \sin (\cdot) \\
0 & -\sin (\cdot) & \cos (\cdot)
\end{array}\right], \\
R_{y}(\cdot): & =\left[\begin{array}{ccc}
\cos (\cdot) & 0 & -\sin (\cdot) \\
0 & 1 & 0 \\
\sin (\cdot) & 0 & \cos (\cdot)
\end{array}\right] .
\end{aligned}
$$

The advantage of using quaternions instead of the matrices from sensors approach, as stated by (34), to determine the tools attitude in order to transform from the drill frame to the earth frame is that when drilling parallel to the earth's magnetic field, the fact the radial magnetometer signals $\mathbf{b}_{z}^{B}$ and $\mathbf{b}_{y}^{B}$ are zero does not prevent the tool attitude from being evaluated. The same problem arises for the matrices from sensors approach when drilling parallel to the earth's gravitational field in that the tool attitude cannot be evaluated, where as with the quaternion approach it can.

\subsection{Euler to Quaternion}

Given the target inclination and azimuth as Euler angles the corresponding quaternion can be found from the relationship

$$
q_{E 2 Q}=\left(\cos \frac{\theta_{i n c}}{2}+\sin \frac{\theta_{i n c}}{2} \mathbf{j}\right)\left(\cos \frac{\theta_{a z i}}{2}+\sin \frac{\theta_{a z i}}{2} \mathbf{i}\right) .
$$


This relationship will be used in order to convert a set point attitude given in terms of azimuth and inclination into a quaternion and hence a vector in the earths frame. The required azimuth and inclination in the inertial reference frame is given by

$$
\mathbf{x}_{d}=q_{E 2 Q}^{-1} \mathbf{r}^{G} q_{E 2 Q}
$$

This defines the attitude as a vector given by a transformation being a rotation about an axis.

\subsection{Output Signals}

For the effective operation of the drill, it is necessary for the directional drillers to monitor the BHA attitude. The practice in the industry is for these to be presented as the the azimuth $\theta_{a z i}$ and inclination $\theta_{\text {inc }}$ angles. These can be calculated from the inclination unit vector by

$$
\begin{aligned}
& \theta_{i n c}=\arccos \frac{\mathbf{x}_{1}}{\|\mathbf{x}\|}, \\
& \theta_{a z i}=\arctan \frac{-\mathbf{x}_{3}}{\mathbf{x}_{2}} .
\end{aligned}
$$

\subsection{Control Signal}

Directional drills will generally have an internal tool face control system. The tool face angle (see Figure 2 ) is the clockwise difference in angle between the projection of $\mathbf{a}$ in the tool face plane and the steering direction in this plane. The tool face angles are determined from the control, $\omega$, by

$$
\theta_{t f}^{\mathbf{a}}=\left\{\begin{array}{l}
\frac{3 \pi}{2}-\arccos \left(\frac{\omega \cdot(\mathbf{a} \times \mathbf{x})}{\|\omega\|\|\mathbf{a} \times \mathbf{x}\|}\right) \text { for } \omega \cdot(\mathbf{a} \times \mathbf{x})>0 \\
\frac{\pi}{2}-\arccos \left(\frac{\omega \cdot(\mathbf{a} \times \mathbf{x})}{\|\omega\|\|\mathbf{a} \times \mathbf{x}\|}\right) \text { for } \omega \cdot(\mathbf{a} \times \mathbf{x})<0 \\
\pi \text { for } \omega \cdot(\mathbf{a} \times \mathbf{x})=0 \text { and } \mathbf{a} \cdot \omega-(\mathbf{a} \cdot \mathbf{x})(\omega \cdot \mathbf{x})>0 \\
0 \text { for } \omega \cdot(\mathbf{a} \times \mathbf{x})=0 \text { and } \mathbf{a} \cdot \omega-(\mathbf{a} \cdot \mathbf{x})(\omega \cdot \mathbf{x})<0
\end{array}\right.
$$

where $\mathbf{a}$ is either $-\mathbf{r}^{G}$ for the case of gravity tool face or $\mathbf{r}^{B}$ for magnetic tool face.

\subsection{Direct calculation of tool-face angle}

The required tool-face angle to implement the control laws proposed in Section 3 can actually be calculated directly without requiring the coordinate transformations listed above, this reduces the computational requirements. It is done by evaluating the $\theta_{t f}^{\mathbf{a}}$ that is in line with the projection of the demand attitude onto a plane perpendicular to the tool as follows:

$$
\begin{aligned}
& \theta_{t f}^{\mathbf{a}}=\theta_{g}-\theta_{r}+2 \pi, \\
& \theta_{t f}^{\mathbf{a}}=\theta_{m}-\theta_{r}+2 \pi,
\end{aligned}
$$


where equations 42 and 43 are $\theta_{t f}^{\text {a }}$ in $G T F$ and $M T F$ respectively, with:

$$
\begin{aligned}
\theta_{r} & =\operatorname{atan} 2\left(-\mathbf{b}_{z}^{d}, \mathbf{b}_{y}^{d}\right), \\
\theta_{g} & =\operatorname{atan} 2\left(-\mathbf{b}_{z}^{G}, \mathbf{b}_{y}^{G}\right), \\
\theta_{m} & =\operatorname{atan} 2\left(-\mathbf{b}_{z}^{B}, \mathbf{b}_{y}^{B}\right), \\
\mathbf{b}^{d} & =R_{y}\left(\theta_{y}\right) R_{x}\left(\theta_{x}\right) \mathbf{x}_{d},
\end{aligned}
$$

and where $R_{x}(\cdot)$ and $R_{y}(\cdot)$ are defined by (35) and (36) respectively and the Euler angles $\theta_{x}$ and $\theta_{y}$ are obtained from spherical to Cartesian followed by Cartesian to spherical transformations (using appropriate sign conventions) of $\theta_{i n c}$ and $\theta_{a z i}$. These transformations are required in order to remove the ambiguity in the axis and sign of angular rotation for $\theta_{i n c}$ as a Euler angle.

\section{Simulation Results}

To demonstrate the effectiveness of the proposed controllers, simulations of the three control laws with the dynamics of (1) are performed. The values of the control gain for the control law defined by (4) is set to $k=5.4^{\circ} \mathrm{hr}^{-1}$ and the constant build rate for the other two control laws is taken as $K=15^{\circ} \mathrm{hr}^{-1}$. The sampling time for the discrete-time controller (15) is $\delta t=100 \mathrm{~s}$. The initial attitude in the earth frame is $\mathbf{x}_{0}=[1,1,0]$, and the attitude demand vector is $\mathbf{x}_{d}=[1,0,0]$.

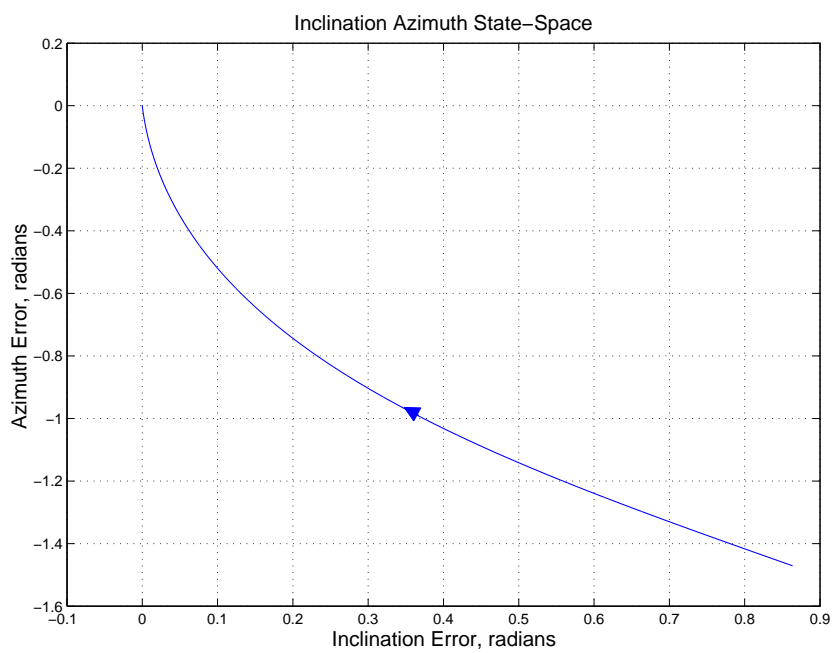

Figure 4: $\theta_{i n c}$ and $\theta_{a z i}$ angle error for the variable build rate controller

Figure 4 shows the attitude trajectory for control law (4) in terms of the inclination angle error and the azimuthal angle error for the unconstrained actuation model for the drill. The curve shown is a state-space trajectory which converges towards $(0,0)$ and remains there.

Figure 5 shows the norm of the attitude error given by $\left\|\mathbf{x}-\mathbf{x}_{d}\right\|$ as a function of time. The error converges asymptotically to zero. 


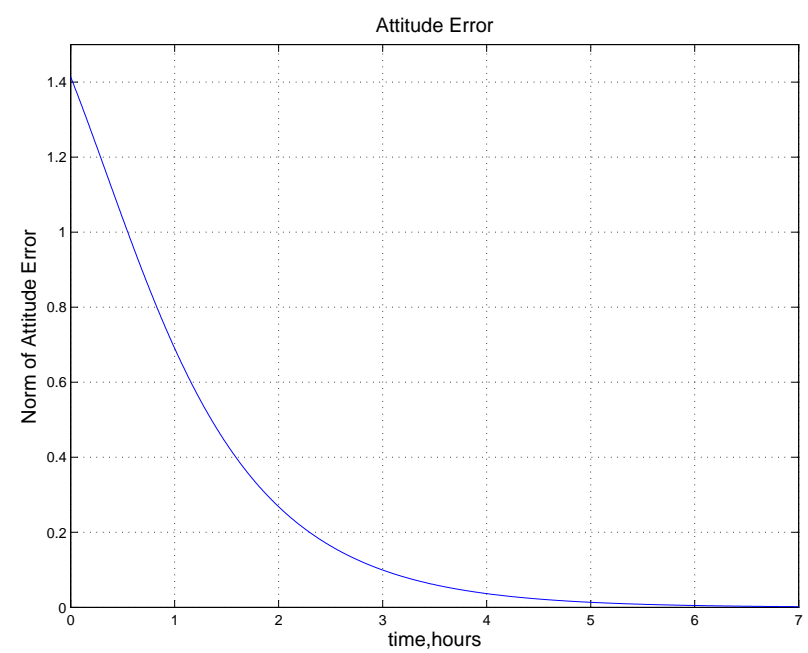

Figure 5: Norm of attitude error for the variable build rate controller

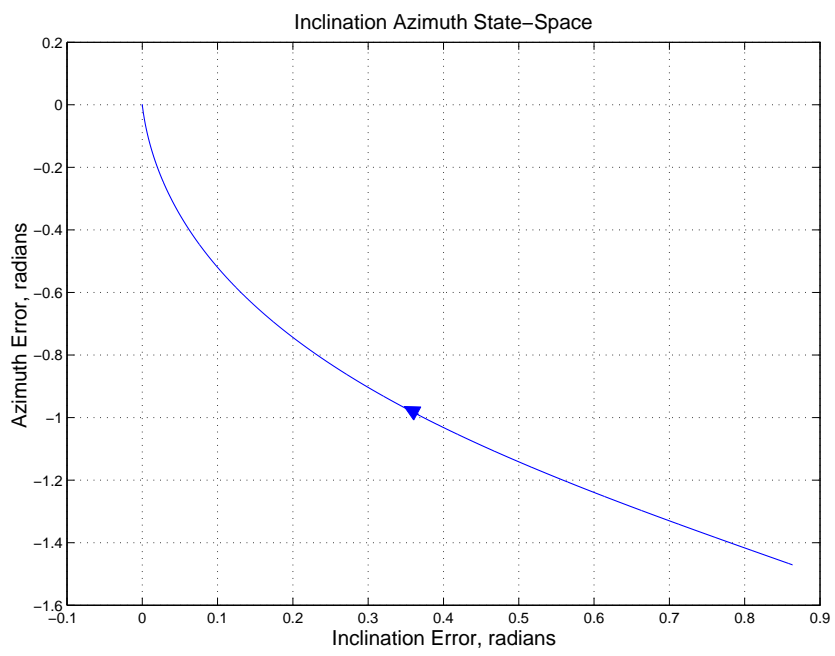

Figure 6: $\theta_{i n c}$ and $\theta_{a z i}$ angle error for the constant build rate controller

Figure 6 shows the attitude trajectory for control law (9). The curve appears identical Figure 4, the trajectory for the variable build rate controller. The path taken by the two trajectories is actually identical, but the speeds along the path differ.

Figure 7 shows the norm of the attitude error given by $\left\|\mathbf{x}-\mathbf{x}_{d}\right\|$ as a function of time. The constant build rate controller converges directly to zero within 6 hours. Although the variable rate controller error converges in approximately the same time, the maximum build rate is much higher than the constant build rate.

Figures 8 and 9 show the attitude trajectory and error for the discrete-time control law (15). The curves are almost identical to Figures 6 and 7 except when the error approaches the bound. Detail of the 


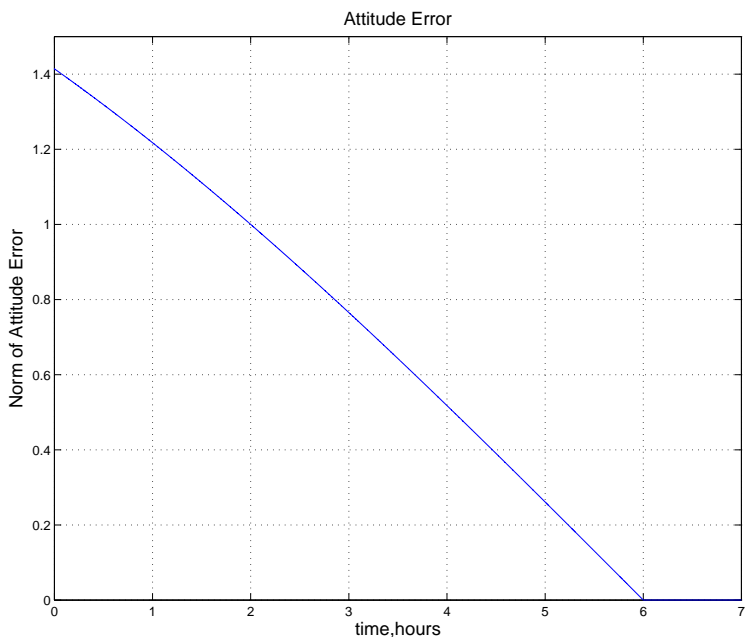

Figure 7: Norm of attitude error for the constant build rate controller

norm of the attitude error plot is shown in Figure 10. The upper bound of the switching region given by $2 \sin \left(K T_{s} / 2\right)$ is also shown. Detail of the error curve near the origin is shown in Figure 11 which shows the discrete switching about the equilibrium point within the upper bound.

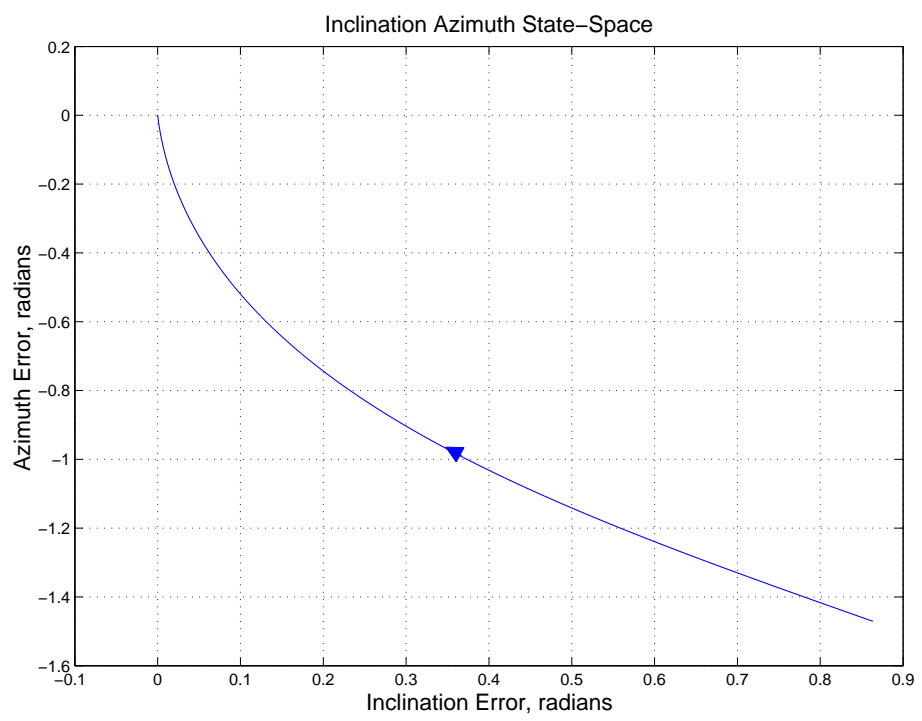

Figure 8: $\theta_{i n c}$ and $\theta_{a z i}$ angle error for the discrete-time controller 


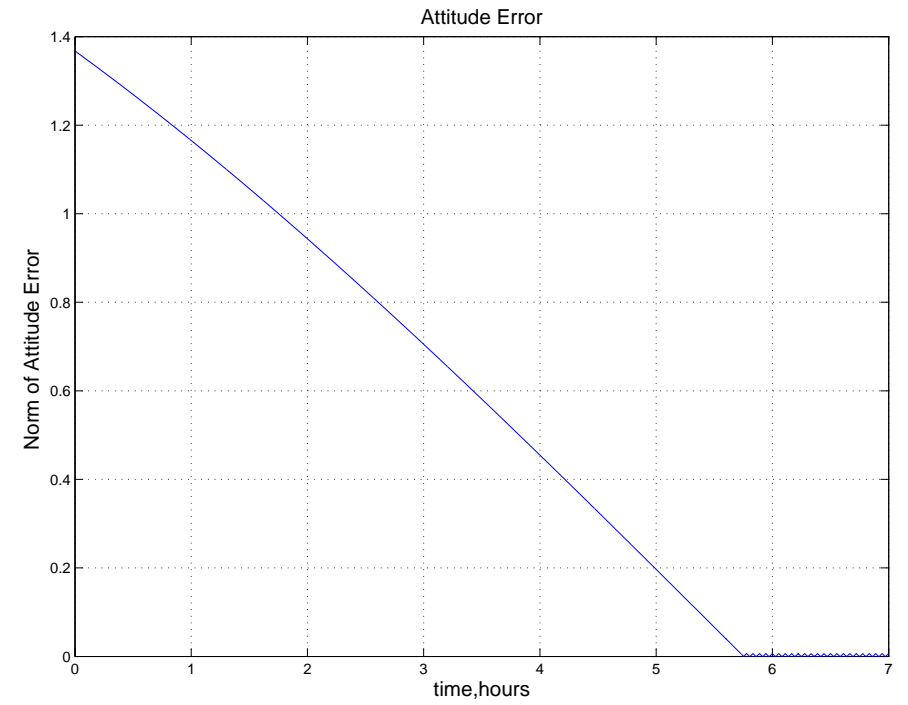

Figure 9: Norm of attitude error for the discrete-time controller

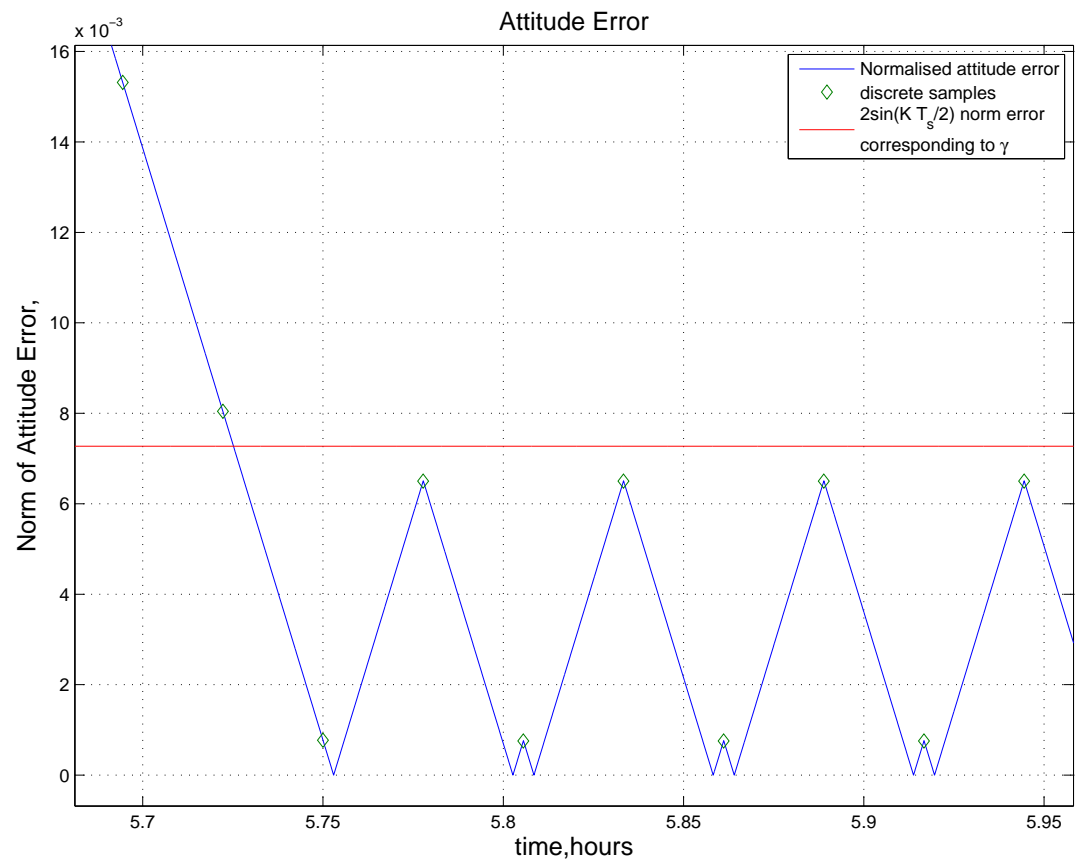

Figure 10: Norm of attitude error for the discrete-time controller - detail near the equilibrium point 


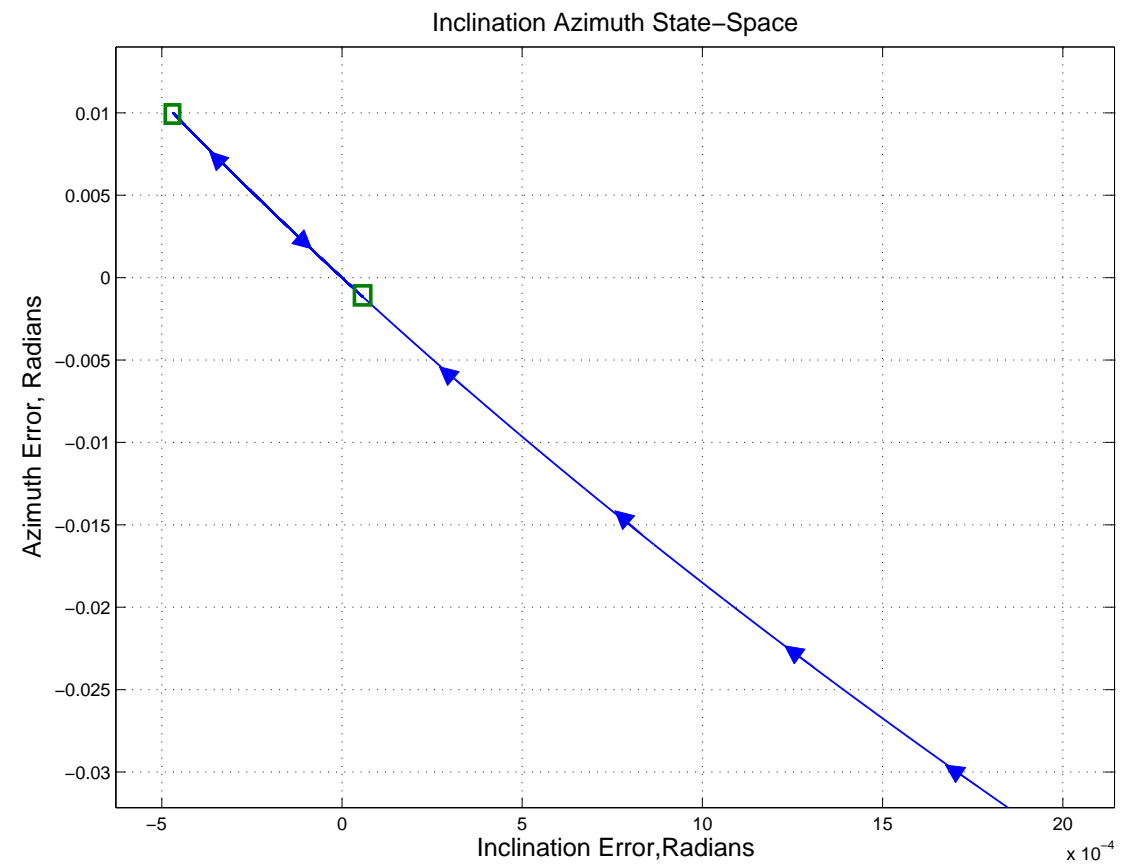

Figure 11: $\theta_{i n c}$ and $\theta_{a z i}$ angle error for the discrete-time controller - detail showing discrete switching near the equilibrium point 


\section{Conclusion}

The paper proposes several control laws for the attitude control of the BHA suitable for directional drilling. By representing the attitude as a unit vector, rather than in terms of the Euler angles, the control laws are simple and elegant. The stability of the controlled system is proven using the Lyapunov direct method. The paper summarizes the relevant quaternion algebra and states a Euler angle based implementation equivalent to the discrete angular velocity vector control law implementation also given in the paper. Simple simulations demonstrate the effectiveness of the proposed controllers. In further work, the control laws will also be tested by more complete simulation subjecting the system to disturbances, measurement errors actuator dynamics and uncertainty. 


\section{References}

[1] S. Williams, "Geosteering: Where are we? Where are we going," in EAGE Geosteering and Well Placement Workshop: Balancing Value and Risk, Dubai UAE, Nov. 2010, keynote address.

[2] S. Jiang, X. Wang, L. Cao, and K. Liu, "A new method for designing 3D trajectory in sidetracking horizontal wells under multi-constraints," in Proc. SPE Asia Pacific Improved Oil Recovery Conference, no. 57282-MS, Kuala Lumpur, Malaysia, Oct. 1999, 8 pages.

[3] A. Li, E. Feng, and Z. Gong, "An optimal control model and algorithm for the deviated well's trajectory planning," Applied Mathematical Modelling, vol. 33, no. 7, pp. 3068-3075, 2009.

[4] R. Baker, A Primer of Oilwell Drilling: A Basic Text of Oil and Gas Drilling, 6th ed. Austin: Univ. of Texas at Austin Petroleum, 2000.

[5] T. Yonezawa, E. J. Cargill, T. M. Gaynor, J. R. Hardin Jr., R. T. Hay, and A. Ikeda, "Robotic controlled drilling: A new rotary steerable system for the oil and gas industry," in Proc. IADC/SPE Drilling Conference, no. 74458-MS, Dallas, TX, Feb. 2002, 15 pages.

[6] S. Kuwana, Y. Kiyosawa, and A. Ikeda, "Attitude control device and drillingdirection control device," U.S. Patent 5316090, May 31, 1994. [Online]. Available: http://www.freepatentsonline.com/5316090.html

[7] N. Panchal, M. T. Bayliss, and J. F. Whidborne, "Robust linear feedback control of attitude for directional drilling tools," in Proc. 13th IFAC Symposium on Automation in Mining, Mineral and Metal Processing, Cape Town, Aug. 2010.

[8] J.-M. Genevois, J. Boulet, C. Simon, and C. Reullon, "Gyrostab Project : The missing link azimuth and inclination mastered with new principles for standard rotary BHAs." in SPE/IADC Drilling Conference, no. 79915-MS, Amsterdam, Netherlands, Feb. 2003, 11 pages.

[9] J. T.-Y. Wen and K. Kreutz-Delgado, "The attitude control problem," IEEE Trans. Automat. Contr., vol. 36, no. 10, pp. 1148-1162, 1991.

[10] J.-J. E. Slotine and W. Li, Applied Nonlinear Control. Englewood Cliffs, NJ: Prentice Hall, 1991.

[11] A. Pressley, Elementary Differential Geometry. London: Springer-Verlag London, 2001.

[12] C. Edwards and C. K. Spurgeon, Sliding Mode Control Theory and Applications. London, UK: T aylor \& Francis, 1998.

[13] F. Fagnani and S. Zampieri, "Stability analysis and synthesis for scalar linear systems with a quantized feedback," IEEE Trans. Automat. Contr., vol. 48, no. 9, pp. 1569-1584, 2003.

[14] F. L. Markley, "Fast quaternion attitude estimation from two vector measurements," Journal of Guidance, Control and Dynamics, vol. 25, no. 2, pp. 411-414, 2002. 